\title{
The use of Stokes-Mueller polarimetry for assessment of amyloid- $\beta$ progression in a mouse model of Alzheimer's disease
}

Borovkova, Mariia, Bykov, Alexander, Popov, Alexey, Pierangelo, Angelo, Novikova, Tatiana, et al.

Mariia Borovkova, Alexander Bykov, Alexey Popov, Angelo Pierangelo, Tatiana Novikova, Jens Pahnke, Igor Meglinski, "The use of Stokes-Mueller polarimetry for assessment of amyloid- $\beta$ progression in a mouse model of Alzheimer's disease," Proc. SPIE 11234, Optical Biopsy XVIII: Toward RealTime Spectroscopic Imaging and Diagnosis, 112340M (21 February 2020); doi: $10.1117 / 12.2550795$

SPIE. Event: SPIE BiOS, 2020, San Francisco, California, United States 


\title{
The use of Stokes-Mueller polarimetry for assessment of amyloid- $\beta$ progression in a mouse model of Alzheimer's disease
}

\author{
Mariia Borovkova*a, Alexander Bykov ${ }^{\mathrm{a}}$, Alexey Popov ${ }^{\mathrm{a}}$, Angelo Pierangelo ${ }^{\mathrm{b}}$, \\ Tatiana Novikova ${ }^{\mathrm{b}}$, Jens Pahnke ${ }^{\mathrm{c}, \mathrm{d}, \mathrm{e}}$, and Igor Meglinski ${ }^{\mathrm{a}, \mathrm{f,g}}$ \\ ${ }^{a}$ Optoelectronics and Measurement Techniques unit, University of Oulu, Oulu 90570, Finland \\ ${ }^{\mathrm{b}}$ LPICM, Ecole polytechnique, Route de Saclay, Palaiseau 91128, France \\ ${ }^{c}$ Department of Neuro-/Pathology, University of Oslo \& Oslo University Hospital, Oslo, Norway \\ ${ }^{d}$ LIED, University of Lübeck, Ratzeburger Allee 160, 23562 Lübeck, Germany \\ e Department of Pharmacology, Medical Faculty, University of Latvia, LV-1004 Riga, Latvia \\ ${ }^{\mathrm{f}}$ School of Engineering and Applied Science, Aston University, Aston St, Birmingham B4 7ET, UK \\ ${ }^{\mathrm{g}}$ School of Life and Health Sciences, Aston University, Aston St, Birmingham B4 7ET, UK
}

\begin{abstract}
Alzheimer's disease, being a major societal burden, demands improvement of current techniques for its treatment and diagnostics. Currently only autopsy histology is able to provide the definite diagnosis for Alzheimer's disease. However, the procedure is rather time consuming and costly. In the current study, we utilized Stokes and Mueller polarimetry techniques to screen for amyloid- $\beta(A \beta)$ deposits in formalin-fixed, paraffin-embedded mouse brain tissue at different stages of Alzheimer's disease. The study has shown that the presence of A $\beta$ plaques influences the properties of scattered polarized light. The Poincaré sphere was used as a graphical tool for the visualization of the alterations of the Stokes vector, obtained with Stokes polarimetry, whereas statistical moments were used for the analysis of depolarization distributions that were acquired with Mueller polarimetry. We demonstrate the sensitivity of the last component of the Stokes vector, the degree of polarization and high-order statistical moments of depolarization to the structural alterations in brain tissue, which correspond to the disease progression. The described approach has a potential to improve the existing pathology screening methods and facilitates $\mathrm{A} \beta$ detection in $\mathrm{AD}$ research.
\end{abstract}

Keywords: depolarization, scattering, anisotropy, statistical analysis, amyloid- $\beta$ plaque, Alzheimer's

\section{INTRODUCTION}

Due to the aging of the population, Alzheimer's disease (AD) has become a major social and financial challenge in the modern world ${ }^{1}$. Presently, conventional imaging techniques, such as magnetic resonance imaging (MRI) and positron emission tomography (PET) are used for the clinical screening of AD patients, but they are limited in spatial resolution and specificity ${ }^{2}$. The definite diagnosis of AD can only be given by histological analysis of post mortem brain tissue. The current routine procedure for the histological analysis in neuropathologic labs uses a number of laborious sample preparation steps, which include fixation of the patient's brain for 2-3 weeks in $4 \%$ formalin, sectioning of the brain, processing and embedding in paraffin of tissue specimens, sectioning with a microtome, mounting on glass slides, staining (immunohistochemical and silver stains for amyloid plaques and tau tangles), and finally microscopy investigation ${ }^{3}$. This procedure is very laborious, time-consuming and strongly relies on the neuropathologist's expertise.

In this study, we apply label-free, polarimetric techniques for the non-invasive analysis of standard paraffin-embedded brain tissue samples (paraffin blocks) of mice at different stages of $\beta$-amyloidosis.

* Correspondence: mariia.borovkova@oulu.fi

Optical Biopsy XVIII: Toward Real-Time Spectroscopic Imaging and Diagnosis, edited by Robert R. Alfano, Stavros G. Demos, Angela B. Seddon, Proc. of SPIE Vol. 11234

$112340 \mathrm{M} \cdot$ - C $2020 \mathrm{SPIE} \cdot \mathrm{CCC}$ code: $1605-7422 / 20 / \$ 21 \cdot$ doi: 10.1117/12.2550795 


\section{METHODS AND MATERIALS}

\subsection{Standard paraffin-embedded blocks of mouse brain tissue}

In the current study, the surface of blocks of formalin-fixed, paraffin-embedded mouse brain tissue (FFPE) at different stages of $\beta$-amyloidosis were analyzed. The hemispheres were excised from sacrificed APP-transgenic mice (APPPS-21, APPtg) ${ }^{4}$ at different ages: 50,75, 100,125, 150, 175, and 200 days. The stage of the $\beta$-amyloidosis correlates with the age of animals. The animals start with $A \beta$ deposition at an age of 45 days.

Figure 1 shows microscopic images of stained histological slides (hemispheres) of three mouse brain samples at an age of (a) 50 days, (b) 125 days, and (c) 200 days. Red arrows in Figure 1(b, c) point at amyloid plaques, one of the major hallmarks of $\mathrm{AD}$, which are observed as dark brownish spots when stained with IHC techniques ${ }^{4-8}$. The increased number of $\mathrm{A} \beta$ plaques correlates with a more advanced stage of the disease.

(a)
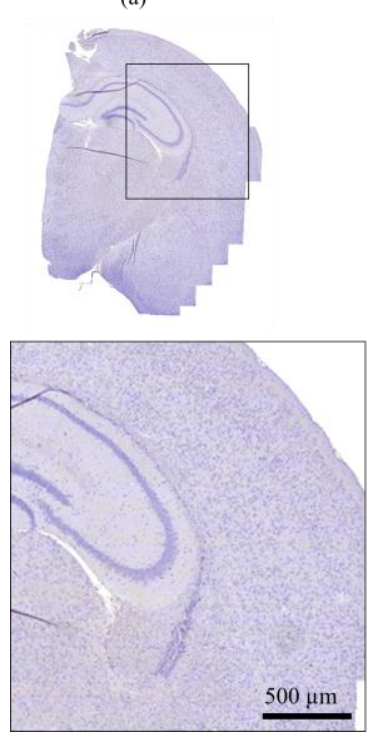

(b)
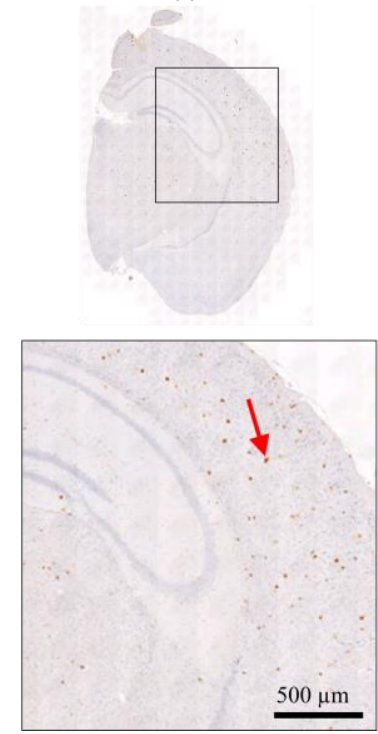

(c)
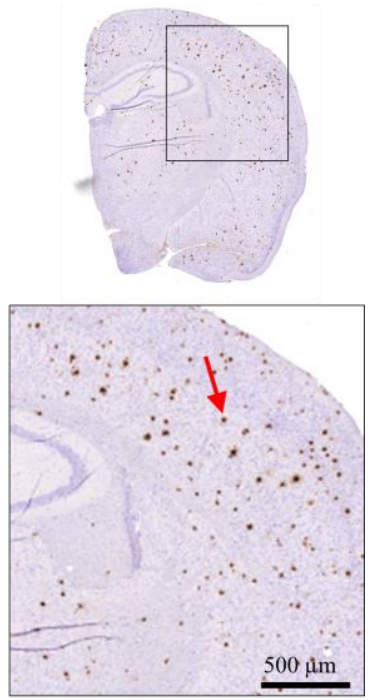

Figure 1. Brain hemisphere of APPtg mice with $\beta$-amyloidosis. Mouse brains were stained with antibody clone 4G8 to highlight $\beta$-amyloid deposits at different stages of AD correlated with the age of a mouse: (a) 50 days, (b) 125 days, and (c) 200 days.

\subsection{Experimental systems}

The principle scheme of the Stokes polarimeter developed in-house ${ }^{9}$ is shown in Figure 2 (a). In the system, the laser light source produced linearly polarized light at $450 \mathrm{~nm}$, then half- and quarter-wave plates transformed the linear polarization of light into right circular polarization. The objective lens focused circularly polarized laser beam on a sample surface (beam diameter was approximately $15 \mu \mathrm{m}$ ). The paraffin-embedded, 4-mm thick slides of mouse brain tissue were placed flat on the 2D scanning stage. Light incident on a sample surface was scattered within the tissue sampling volume, which was adjusted by the variation of the distance of detection (Fig. 2(a)). Light backscattered from the sample was collected with an objective lens, spatially filtered by a diaphragm and analyzed by the Stokes polarimeter, which consists of a rotating quarter-wave plate, polarizer and powermeter. The angles of incidence and detection were 55o and 30o, respectively; this was optimized in order to eliminate specular reflection in the detected signal. The scanning of the samples was performed over $5 \times 5 \mathrm{~mm} 2$ area with a $25-\mu \mathrm{m}$ step. The acquired distributions of Stokes vector were averaged over the scanning area. The principle scheme of the multi-wavelength wide field Mueller imaging polarimeter, developed in LPICM, Ecole polytechnique10,11, is shown in Figure 2(b).

The state of polarization of the incident white light was sequentially transformed by the polarization stage generator (PSG) into four different polarization states. For each generated polarization state of incident light, the light scattered back from the sample was analyzed by the polarization state analyzer (PSA), which filtered sequentially the same four polarization states, as the ones generated by the PSG. Both the PSG and PSA are assembled from linear polarizer and two liquid crystals, 
the order of optical elements is reversed for PSA with respect to PSG. The light filtered by the PSA passed through a 40 $\mathrm{nm}$ wide interference filter, centered at $450 \mathrm{~nm}$, and was recorded by the CCD camera. Thus, 16 intensity images were recorded and processed with regard to the instrument calibration data, resulting into the Mueller matrix which contains full polarimetric response of the examined sample. The field of view of the system was $19 \times 9 \mathrm{~mm} 2$ with $0.4 \mathrm{~mm}$ spatial resolution.

(a)

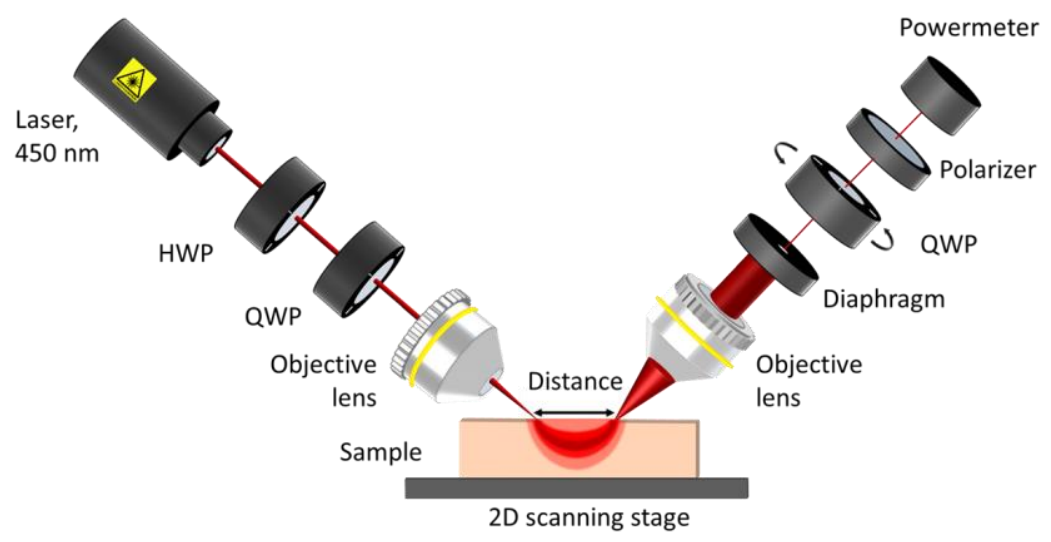

(b)

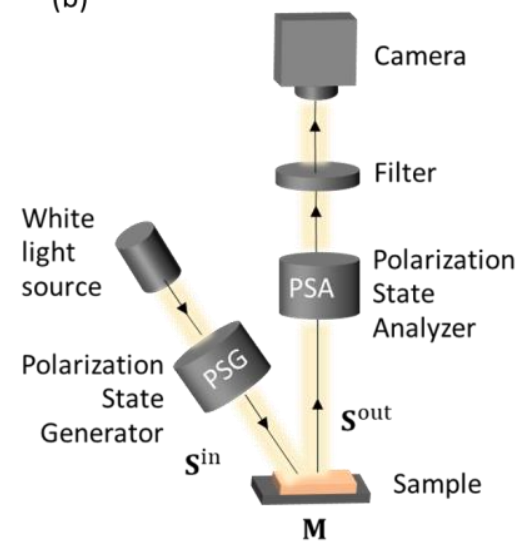

Figure 2. Schematic layout of experimental systems: (a) Stokes polarimeter, (b) Mueller imaging polarimeter. Explanations are given in the text.

\section{RESULTS AND DISCUSSION}

The experimental results of the screening of 7 FFPE tissue blocks of mouse brain samples at different stages of the AD are shown in Figure 3: (a) degree of polarization in \%, (b) V component of Stokes vector; (c) positions of Stokes vector mapped into the Poincaré sphere with respect to the degree of polarization.

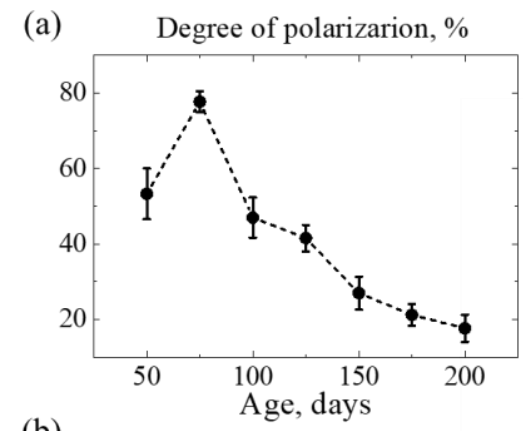

(b)

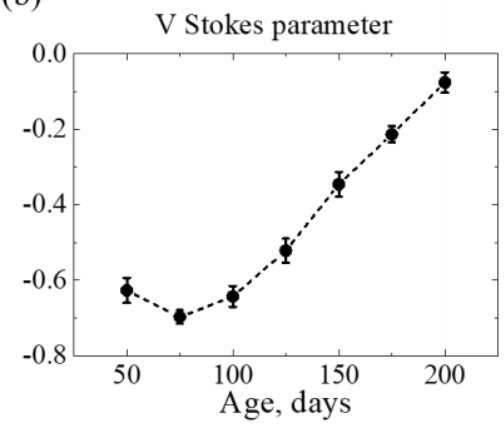

(c)

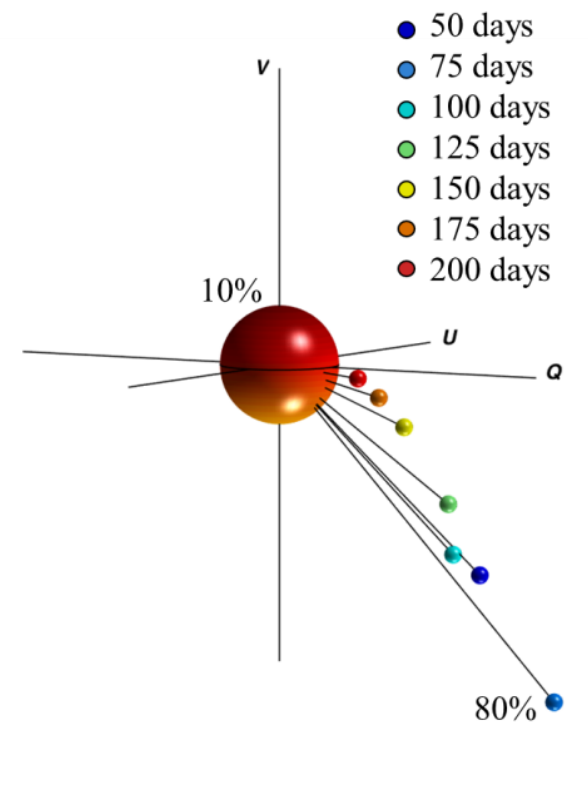

Figure 3. Change of the Stokes vector with $\beta$-amyloidosis progression (from 50 to 200 days): (a) degree of polarization in $\%$, (b) V Stokes parameter, (c) Stokes vector mapped on the Poincaré sphere with respect to degree of polarization. The radius of the sphere corresponds to $10 \%$ of degree of polarization. The highest magnitude of Stokes vector degree of polarization shown in figure is $80 \%$. 
According to the experimental results shown in Figure 3, alterations of the brain tissue, associated with the progression of the disease, affect the degree of polarization and the relative phase shift between transverse components of electric field of polarized light, which is observed as a translation of the Stokes vector towards positive hemisphere of the Poincaré sphere and inside the sphere. Due to the increase in scattering of brain tissue, conditioned by the accumulation of amyloid plaques (this has been demonstrated in literature ${ }^{12}$ ) the degree of polarization decreases and the phase shift between electric field components changes gradually with the disease progression.

The typical Mueller matrix images of the mouse brain tissue samples obtained with the Mueller polarimeter are presented in Figure 4 (a). The matrices were diagonal for all 21 measured samples ( 3 samples for each of 7 stages of the disease), which means that paraffin-embedded blocks of mouse brain tissue acted as a partial depolarizer and did not exhibit any anisotropy of amplitude or phase. The spatial distributions of total depolarization parameter $\Delta=1-\frac{m 22+m 33+m 44}{3}$ were analyzed using statistical moments of $1^{\text {st }}$ to $4^{\text {th }}$ order, as it was done in previously published works ${ }^{13,14}$. The obtained trends of the $1^{\text {st }}$ to $4^{\text {th }}$ statistical moments (mean value, standard deviation, skewness and kurtosis) are shown in Figure 4 (b).

(a)

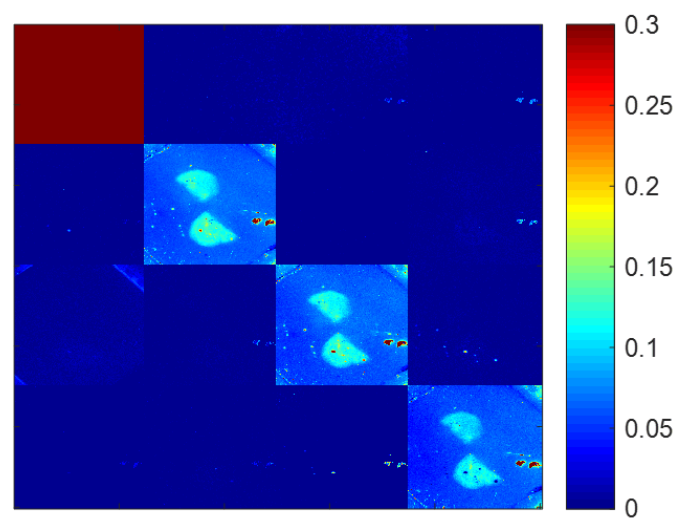

(b)

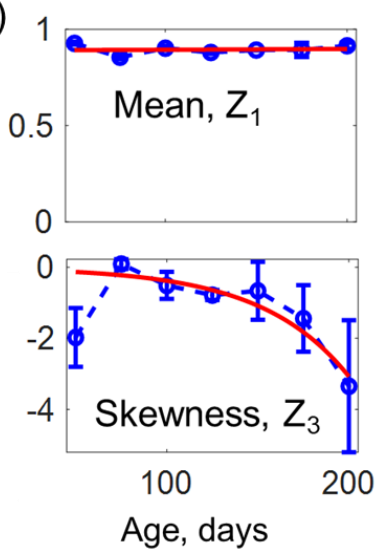

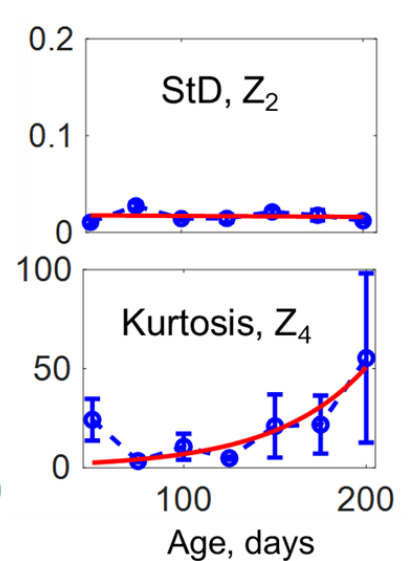

Figure 4. Experimental results obtained with the Mueller polarimetry: (a) typical Mueller matrix of a mouse brain sample; (b) Statistical moments of $1^{\text {st }}$ to $4^{\text {th }}$ order (mean, standard deviation, skewness, kurtosis). Blue points represent the experimentally obtained data, red lines show the results of the fit: linear for $1^{\text {st }}$ and $2^{\text {nd }}$ moments and exponential for $3^{\text {rd }}$ and $4^{\text {th }}$ moments.

According to the obtained results, the statistical moments of the $1^{\text {st }}$ and $2^{\text {nd }}$ order of the distributions of total depolarization at different stages of the disease do not exhibit noticeable changes with the disease progression. Due to the limited resolution of the instrument and different optical properties of brain areas ${ }^{12}$, differently affected by the disease, $1^{\text {st }}$ and $2^{\text {nd }}$ statistical moments are not sensitive to the disease progression. However, $3^{\text {rd }}$ and $4^{\text {th }}$ moments show significant changes, approximated by the exponential fit. This indicates that the distribution of depolarization values is becoming less symmetrical and more peaked with weaker outliers with more severe stage of the disease. This is explained by the change in anisotropy of scattering conditioned by the increase of the scatterers' size ${ }^{12}$ due to accumulation of neuronal plaques. With the later stage of the disease, the angular dependence of scattering is growing, and the sampling distribution of depolarization values becomes deviated from normal, therefore, the results show the increased absolute skewness and kurtosis.

\section{CONCLUSIONS}

In the current study, we demonstrate the applicability of polarization-based techniques for the non-destructive label-free screening of standard paraffin-embedded blocks of mouse brain tissue with the aim of differentiating the AD stage. We have revealed the sensitivity of V parameter of Stokes vector and the degree of polarization, acquired with the laser-based scanning-based approach of Stokes-vector polarimetry with incident circularly polarized light, to the morphological alterations of brain tissue, associated with the progression of AD. Moreover, we have shown the sensitivity of high-order statistical moments of spatial distributions of total depolarization, acquired with Mueller polarimeter to the AD 
progression. The combination of the described optical modalities can potentially improve existing pathology screening techniques, as the described protocols are non-destructive and label-free.

\section{ACKNOWLEDGEMENTS}

This project has received funding from the European Union's Horizon 2020 research and innovation programme under the Marie Skłodowska-Curie grant agreement No.713606, Cost Actions CA16118, the ATTRACT project funded by the EC under Grant Agreement 777222, Academy of Finland (grants: 314369 and 325097), and INFOTECH strategic funding.

The work of J.P. was supported by the following grants: Deutsche Forschungsgemeinschaft/ Germany (DFG PA930/12); Wirtschaftsministerium Sachen-Anhalt (EFRE, ZS/2016/05/78617) and the Leibniz Association (Leibniz-Wettbewerb SAW-2015-IPB-2); Latvian Council of Science FLPP/ Latvia (lzp-2018/1-0275); Nasjonalforeningen (16154), HelseSØ/ Norway (2016062, 2019054, 2019055); Norsk forskningsrådet/ Norway (251290 FRIMEDIO, 260786 PROP-AD); Horizon 2020/ European Union (643417 (PROP-AD)). PROP-AD is an EU Joint Programme - Neurodegenerative Disease Research (JPND) project. The project is supported through the following funding organisations under the aegis of JPND www.jpnd.eu (AKA, no.301228 - Finland, BMBF \#01ED1605- Germany, CSO-MOH \#30000-12631 - Israel, NFR \#260786 - Norway, SRC \#2015-06795 - Sweden). This project has received funding from the European Union's Horizon 2020 research and innovation programme under grant agreement \#643417 (JPco-fuND).

\section{REFERENCES}

[1] Reitz, C. and Mayeux, R., "Alzheimer disease: Epidemiology, diagnostic criteria, risk factors and biomarkers," Biochem. Pharmacol., 88(4), 640-651 (2014).

[2] Choi, E. J., Son, Y. D., Noh, Y., Lee, H., Kim, Y. B. and Park, K. H., "Glucose hypometabolism in hippocampal subdivisions in Alzheimer's disease: A pilot study using high-resolution 18F-FDG PET and 7.0-T MRI," J. Clin. Neurol., 14(2), 158-164 (2018).

[3] Slaoui M., Fiette L., "Histopathology procedures: from tissue sampling to histopathological evaluation," Drug safety evaluation, Humana Press, 69-82 (2011).

[4] Radde R., Bolmont T., Kaeser S.A., Coomaraswamy J., Lindau D., Stoltze L., Calhoun M.E., Jäggi F., Wolburg H., Gengler S., Haass C., "A $\beta 42-$ driven cerebral amyloidosis in transgenic mice reveals early and robust pathology," EMBO reports, 7(9): 940-6 (2006).

[5] Paarmann K., Prakash S.R., Krohn M., Möhle L., Brackhan M., Brüning T., Eiriz I., Pahnke J., "French maritime pine bark treatment decelerates plaque development and improves spatial memory in Alzheimer's disease mice," Phytomedicine, 1;57:39-48 (2019).

[6] Steffen J., Stenzel J., Ibrahim S., Pahnke J., "Short-term effects of microglia-specific mitochondrial dysfunction on amyloidosis in transgenic models of Alzheimer's disease," J. Alzheimer's Dis., 1;65(2):465-74 (2018).

[7] Steffen J., Krohn M., Schwitlick C., Brüning T., Paarmann K., Pietrzik C.U., Biverstål H., Jansone B., Langer O., Pahnke J., "Expression of endogenous mouse APP modulates $\beta$-amyloid deposition in hAPP-transgenic mice," Acta Neuropathol. Commun.; 5(1):49 (2017).

[8] Krohn M., Lange C., Hofrichter J., Scheffler K., Stenzel J., Steffen J., Schumacher T., Brüning T., Plath A.S., Alfen F., Schmidt A., "Cerebral amyloid- $\beta$ proteostasis is regulated by the membrane transport protein ABCC1 in mice," Clin. Drug Investig.;121(10):3924-31 (2011).

[9] Borovkova M., Bykov A., Popov A., Meglinski I., "Influence of scattering and birefringence on the phase shift between electric field components of polarized light propagated through biological tissues," European Conference on Biomedical Optics, Opt. Soc. Am., p. 11075_25 (2019).

[10] Novikova T., Rehbinder J., Deby S., Haddad H., Vizet J., Pierangelo A., Validire P., Benali A., Gayet B., Teig B., Nazac A., "Multi-spectral Mueller matrix imaging polarimetry for studies of human tissues," Clinical and Translational Biophotonics, Opt. Soc. Am., TTh3B-2 (2016).

[11] Agarwal N., Yoon J., Garcia-Caurel E., Novikova T., Vanel J.C., Pierangelo A., Bykov A., Popov A., Meglinski I., Ossikovski R., "Spatial evolution of depolarization in homogeneous turbid media within the differential Mueller matrix formalism," Opt. Lett., 40(23), 5634-7 (2015).

[12] Lee M., Lee E., Jung J., Yu H., Kim K., Yoon J., Lee S., Jeong Y., Park Y.,'Label-free optical quantification of structural alterations in Alzheimer's disease," Sci. Rep., 6:31034 (2016). 
[13] Borovkova M., Peyvasteh M., Dubolazov O., Ushenko Y., Ushenko V., Bykov A., Deby S., Rehbinder J., Novikova T., Meglinski I., "Complementary analysis of Mueller-matrix images of optically anisotropic highly scattering biological tissues," J. Eur. Opt. Soc. - Rapid., 14(1):20 (2018).

[14] Borovkova M., Trifonyuk L., Ushenko V., Dubolazov O., Vanchulyak O., Bodnar G., Ushenko Y., Olar O., Ushenko O., Sakhnovskiy M., Bykov A., "Mueller-matrix-based polarization imaging and quantitative assessment of optically anisotropic polycrystalline networks," PloS one, 14(5) (2019). 